\title{
TRANSFORMATION TEXTURE ANALYSIS OF BCC AND BCT FERROUS MARTENSITE
}

\author{
HIROFUMI MIYAJI AND EI-ICHI FURUBAYASHI \\ National Research Institute for Metals, 2-3-12, Nakameguro, Meguro-ku, \\ Tokyo 153, Japan
}

\begin{abstract}
The theoretical prediction of transformation textures, that developed in thin sheet without external stress, was carried out based on the Bain-Strain and Twinning-Shear model, respectively. To examine the validity of the prediction, the experiments were conducted with X-ray pole figure method on $\mathrm{Fe}-30 \mathrm{Ni}$ and $\mathrm{Fe}-30 \mathrm{Ni}-0.8 \mathrm{C}$ alloy sheets. The results obtained are as follows:

(1) Variant selection phenomenon depends on the crystal orientation of the parent phase and on the Bain strain in the martensitic transformation.

(2) The effect of the anisotropic constraint stress on the shear deformation involved in the lattice change and on the Bain distortion appears most remarkably in the BCT martensite transformed from the cube textured parent phase.

(3) It became clear that such a variant that has the largest Bain strain component to the sheet normal, that is, the variant, of which the Bain distortion is prevented least of all by the anisotropic constraint stress, forms predominantly.
\end{abstract}

KEY WORDS: Martensitic transformation, variant selection, Bain strain, transformation texture, iron nickel alloy, constraint stress.

\section{INTRODUCTION}

Variant selection phenomenon during martensitic transformation has been observed in many steels and iron base alloys. Several models for variant selection have been proposed over the past few decades. Among them, two important models which seem to explain the observation results satisfactorily, were proposed recently.

At first Higo, Lecroisey and Mori (1974) pointed out the importance of the initial stage of the transformation from the parent phase to the martensite phase which occurred by the shear deformation of lattice of the small amount of participated atoms. On the transformation mechanism of martensite, the models which are composed of combination of some elementary processes in which one or two shear deformation is concerned, were proposed by Kurdjumov and Sachs (1930), Nishiyama $(1934 ; 1936)$ and Bogers and Burgers (1964). All of them postulate that the shear movement of atoms on $\{111\}$ plane to $\langle 112\rangle$ direction occurs at the beginning of transformation. This shear deformation (strain) will be defined as the "characteristic strain" which is used to calculate the degree of interaction with stress in variant selection models.

In $\gamma$ phase, the crystal possesses 12 twinning shear systems in all. Higo, Lecroisey and Mori (1974) showed that the variant, which formed predominantly, was such that induced by the operation of the active twinning shear system having the maximum 
resolved shear stress with respect to the applied stress. This concept will be called "Twinning Shear (TS) model", since the $\{111\}<112>$ shear coincides with the twinning shear in FCC metals.

On the other hand, Furubayashi (1985a; 1985b) and Furubayashi, Miyaji and Nobuki (1987) remarked to the interaction between the stress and "lattice deformation" in the phenomenological theory of martensitic transformation. Austenite lattice having face centered cubic structure may also be described as a body centered tetragonal structure with an axial ratio of $\sqrt{2}$. Bain (1924) proposed that the martensitic transformation merely involves the deformation of about $20 \%$ compression to the $\mathrm{c}$ axis ([001] direction) and about $12 \%$ expansion to the perpendicular directions of the austenite unit cell. This deformation (strain) is defined as the characteristic strain of transformation. There are three ways of choosing this type of tetragonal lattice in the austenite lattice, that is, the direction of $\mathrm{c}$ axis can be taken parallel to either one of $\mathrm{X}, \mathrm{Y}$ or $\mathrm{Z}$ axis, respectively and Furubayashi $(1985 \mathrm{a} ; 1985 \mathrm{~b})$ considered that the variant, which is selected, is such that the characteristic strain is assisted most effectively by the applied stress (the work done by the stress is maximum). This view will be called "Bain Strain (BS) model" hereafter.

Furubayashi (1985a) carried out the computer simulations of transformation textures formed from warm rolled austenite in order to assess the validity of the various variant selection criteria. He contended that TS and BS models were the most promising since these could account for the experimental transformation textures with reasonable accuracy.

But it seems when the external stress is applied that these models predict the same results (Miyaji and Furubayashi, 1990). In this report it is proved theoretically that these models lead to the different results when the external stress is not applied and/but the anisotropical constraint stress exists. Through the present experiments to verify this, it becomes clear that the BS model is more correct than TS model. The details will be mentioned below in order.

\section{PREDICTION OF VARIANT SELECTION IN THIN SHEET}

\subsection{Effect of anisotropy of constraint stress}

Hashimoto (1981) showed that the variant selection phenomenon occurs in thin sheet of steel even without external stress, because the heterogeneity of internal stress due to constraint from the surrounding parent phase makes a significant effect to the martensitic transformation. It will be abbreviated "constraint stress" hereafter. He thought that the constraint stress against the transformation strain would be weaker in the normal direction of the sheet than in other directions. Accordingly, the deformation occured easily to that direction and the variant, which had the largest deformation component to that direction, formed predominantly.

Liu and Bunge (1991) found by microscopical inspection that the height of surface relief was higher for the variants which were selected and lower for them which were not selected. They considered the reason of it as follows. Lower surface relief means that the shear direction during phase transformation lies nearly parallel to the sheet plane. Hence, the resistance to lattice deformation imposed by neighbouring grains must be larger in this case. Therefore, the variants belonging to this group are not chosen. They called it as "thin-sheet effect." 
The present authors (Miyaji and Furubayashi, 1990; Miyaji and Furubayashi, 1991) verified this fact on the Fe-30Ni alloy having the composition shown in Table 1; both austenite $(\gamma)$ before transformation and martensite $(\alpha)$ after transformation were present at room temperature in this alloy. The $\{001\}$ pole figure of $\gamma$ phase of the Fe-30Ni alloy is shown in Figure 1(a). According to this, the cube texture has been clearly developed in $\gamma$ phase. On the other hand, $\{001\}$ pole figure of $\alpha$ phase transformed without external stress is shown in Figure 1(b). Only the variant $\mathrm{Z}$ component having the Bain compression axis to the normal direction of the sheet is formed as discussed in the next chapter.

At first, the effect of constraint stress on the variant selection is calculated in the case of BS model and TS model. Both of these models postulate that the variant, that is selected, is the one that will lead to the maximum work being done by the stress (applied or developed stress) against the characteristic strain of martensitic transformation (Bain strain or twinning shear strain of the $\gamma$ phase).

Table 1 Chemical composition of alloy (wt \%).

\begin{tabular}{llllllll}
\hline Alloy & $N i$ & $C$ & $N$ & \multicolumn{5}{c}{ Others } \\
\hline Fe-30Ni & 29.67 & 0.001 & 0.001 & Al:0.025 & Mn:0.085 & Si:0.002 & S:0.003 \\
Fe-30Ni-0.8C & 29.67 & 0.80 & 0.01 & Al:0.025 & Mn:0.085 & So:0.002 & S:0.003 \\
\hline
\end{tabular}

(a)

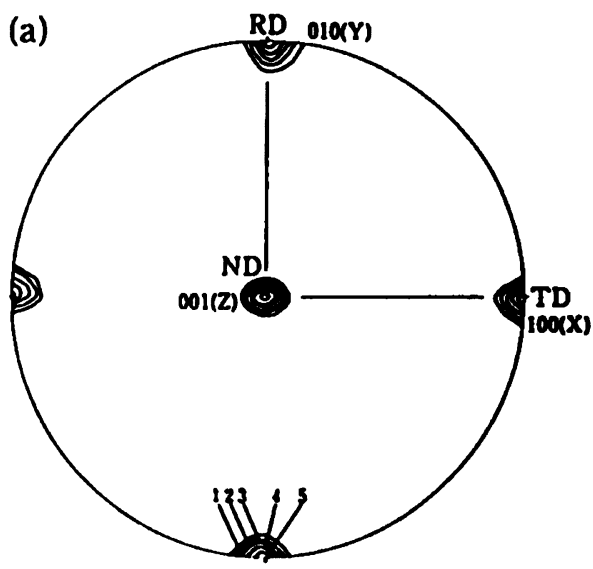

(a) austenite, (b) cubic $\left(\left|\varepsilon_{1}\right|>\left|\varepsilon_{2}\right|\right)$ martensite transformed from cube-textured austenite in (a). Only the variant $Z$ was observed in it.

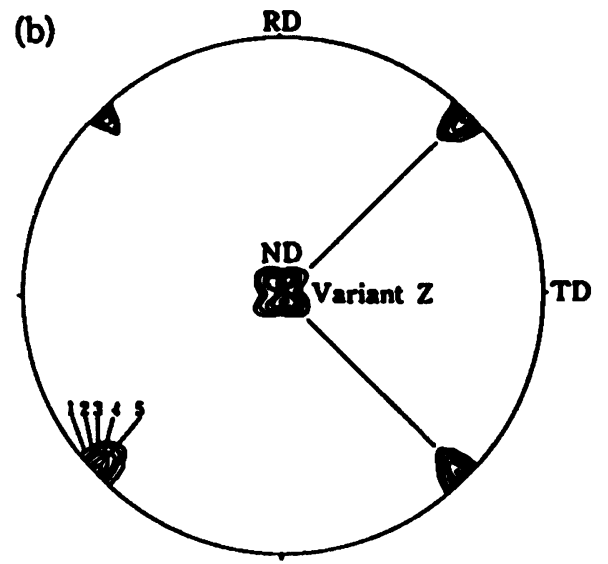

Figure $1\{001\}$ pole figure of $\mathrm{Fe}-30 \mathrm{Ni}$ alloy. 


\subsection{Calculation of the work in BS model}

In a series of our studies, the authors postulated the BS model (Furubayashi, 1985a; Furubayashi, 1985b; Furubayashi, Miyaji and Nobuki, 1987; Miyaji and Furubayashi, 1990; Miyaji and Furubayashi, 1991). In the analysis of the present study, the BainStrain model is also postulated. The Bain strain is denoted by the compressive strain $\varepsilon_{1}$ to the $c$ axis ([001] direction) and expansion strain $\varepsilon_{2}$ to the perpendicular directions of the austenite unit cell. The $\varepsilon_{1}$ and $\varepsilon_{2}$ are described by following equations, using the lattice parameter of $\gamma\left(a_{0}\right)$ and of $\alpha$ (c and a)

$$
\begin{aligned}
& \varepsilon_{1}=\left(c-a_{0}\right) / a_{0} \\
& \varepsilon_{2}=\left\{a-\left(a_{0} / \sqrt{2}\right)\right\} /\left(a_{0} / \sqrt{2}\right)
\end{aligned}
$$

Because the compressive axis (c axis) can be taken parallel to either one of the three axes, three variants (denoted Bain variants hereafter) can be formed by Bain strain. We call them variant $X$, variant $Y$ and variant $Z$, respectively.

Now, when the constraint stress along the normal direction of the sheet diminishes, due to two dimentional sheet shape, by $\Delta \sigma_{N}$, the work W, done by it against the unit volume of the parent phase, decreases by

$$
\Delta \mathrm{W}=\Delta \sigma_{\mathrm{N}} \cos \Theta_{\xi}^{i} \cdot \varepsilon_{1}+\Delta \sigma_{\mathrm{N}} \cos \Theta_{\eta}^{i} \cdot \varepsilon_{2}+\Delta \sigma_{\mathrm{N}} \cos \Theta_{\xi}^{i} \cdot \varepsilon_{2}
$$

where $\Theta_{\xi}^{i}$ is the angle between the Bain compression axis of variant $i$ and normal direction ${ }^{\xi}$ of the sheet, $\Theta_{\eta}^{i}$ and $\Theta_{\xi}^{i}$ are those between Bain expansion axes of variant $i$ and normal direction of the sheet, respectively. $\Delta \sigma_{\mathrm{N}}$ is positive to the compressive strain and negative to the expansive strain, because it acts always to prevent the deformation.

From Eq. 3 it became clear that the value of $\Delta \mathrm{W}$ changes depending on the initial orientation of the sheet $\left(\Theta_{\xi}^{i}, \Theta_{\eta}^{i}, \Theta_{\zeta}^{i}\right)$ and Bain strain component $\left(\varepsilon_{i}, \varepsilon_{2}\right)$.

\subsection{Calculation of the work in TS model}

Twinning shear in the fcc crystal occurs to the shearing direction of the $\left.\{111\}_{\gamma}<112\right\rangle_{\gamma}$ shear system.

Now, when the constraint stress along the normal direction of the sheet diminishes by $\Delta \sigma_{\mathrm{N}}$, the work $\mathrm{W}$ done by the constraint stress decreases by

$$
\Delta \mathrm{W}=\Delta \sigma_{\mathrm{N}} \gamma_{t} \cos \Phi \cdot \cos \lambda
$$

where $\gamma_{t}$ is the characteristic strain in TS model, $\Phi$ is the angle between sheet plane and shear plane and $\lambda$ is the angle between the normal direction of the sheet (direction of the $\Delta \sigma_{\mathrm{N}}$ ) and the shear direction.

In $\gamma$ phase, the crystal possesses 12 twinning shear system in all. Higo, Lecroisey and Mori (1974) showed that the variant induced by the operation of the active shear system, which has the maximum resolved shear stress with respect to the applied stress (that is, the variant which has the nearest compression axis (c axis) to the shear direction of active shear systems), forms predominantly.

It became clear from Eq. 4 that the value of $\Delta W$ changes depending on the initial orientation of the sheet $(\Phi$ and $\lambda$ ). 


\subsection{PREDICTION OF VARIANT SELECTION}

\subsubsection{Effect of initial orientation and Bain strain in BS model}

(1) In the case when $\left|\varepsilon_{1}\right|>\left|\varepsilon_{2}\right|$

The effect of $\gamma$ orientation on the value of $\Delta \mathrm{W}$ of the three Bain variants was calculated according to Eq.(3) in the case of $\varepsilon_{1}=-0.2$ and $\varepsilon_{2}=0.12$ (it is the Bain strain in $\mathrm{fcc} \rightarrow$ bcc transformation of Fe-30Ni alloy (Breedis and Wayman, 1962)). The result was shown on stereographic triangle in Figure 2(a). It became clear from Figure 2(a) that the value of $\Delta \mathrm{W}$ of each variant changes with the $\gamma$ orientations, respectively.
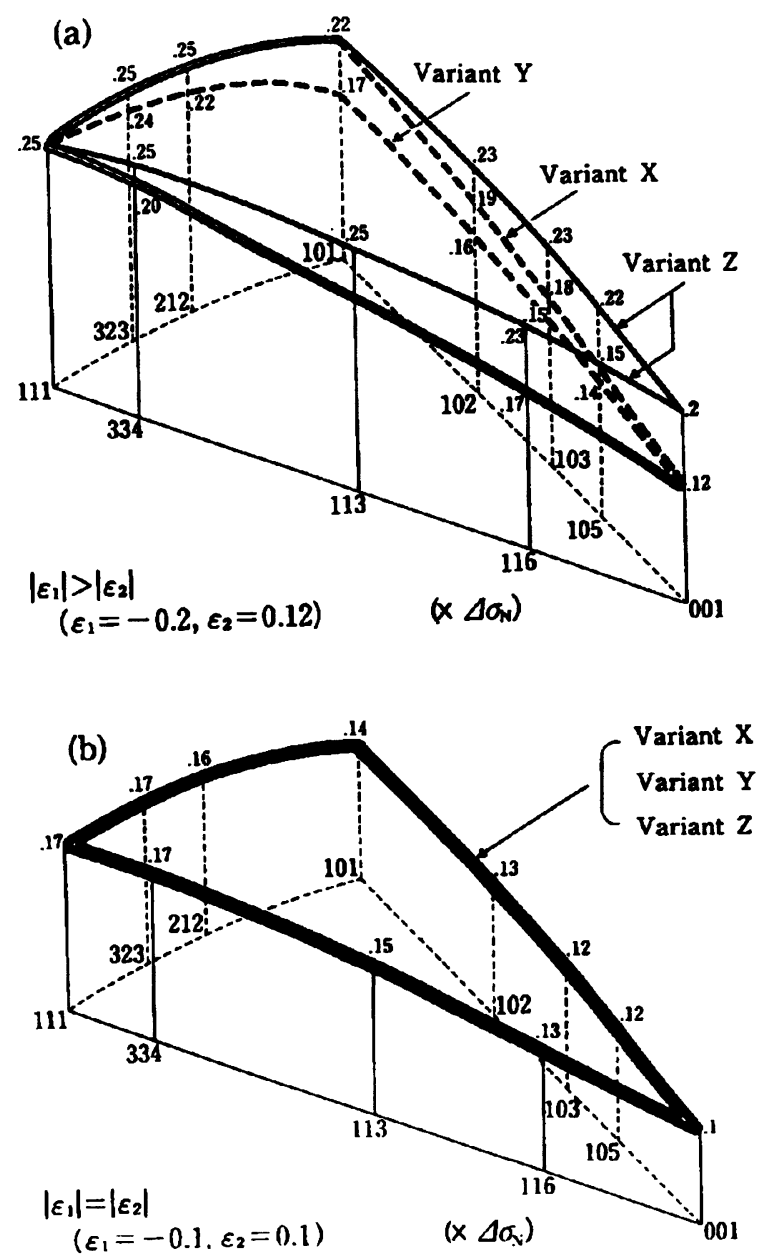

Figure 2 Effect of initial orientations on the work done by the diminished constraint stress against the Bain strain is calculated for each variant, and plotted on the stereographic triangle.

(a) $\left|\varepsilon_{1}\right|>\left|\varepsilon_{2}\right|$, (b) $\left|\varepsilon_{1}\right|=\left|\varepsilon_{2}\right|$. 
In general, as it is considered that the easiness of martensitic transformation is proportional to the value of $\Delta \mathrm{W}$, variant selection occurs more easily in the $\gamma$ orientations in which the differences of these values are more large (Hashimoto, 1981).

It is seen from Figure 2(a) that the difference of them is maximum in $(001)_{\gamma}$ orientation and minimum (zero) in (111) ${ }_{\gamma}$ orientation. Accordingly the variant selection is most probable in $(001)_{\gamma}$ orientation and not at all in $(111)_{\gamma}$ orientation.

(2) In the case when $\left|\varepsilon_{1}\right| \leqq\left|\varepsilon_{2}\right|$

The effect of $\gamma$ orientation on the value of $\Delta \mathrm{W}$ of the three Bain variants was calculated according to Eq. (3) in the case of $\left|\varepsilon_{1}\right|=\left|\varepsilon_{2}\right|\left(\varepsilon_{1}=\angle 0.1, \varepsilon_{2}=0.1\right)$. The result was shown on stereographic triangle in Figure 2(b). It became clear from Figure 2(b) that the value of $\Delta \mathrm{W}$ of each variant changes with the $\gamma$ orientations, respectively. But the differences of them between three variants were not recognized at all. That is, it is considered that the variant selection does not occur at all.

\subsubsection{Effect of initial orientation in TS model}

The effect of $\gamma$ orientation on the value of $\Delta \mathrm{W}$ done by the constraint stress against the shear deformation was calculated according to Eq. 4. As mentioned already in section 2.3., in $\gamma$ phase, the crystal possesses 12 twinning shear systems in all. Variants formed by the activation of the shear systems are divided into 3 groups containing the same kind of 4 variants, respectively (Miyaji and Furubayashi, 1990). One of them which had the largest value of $\Delta \mathrm{W}$ was selected from each groups and shown in stereographic triangle in Figure 3.

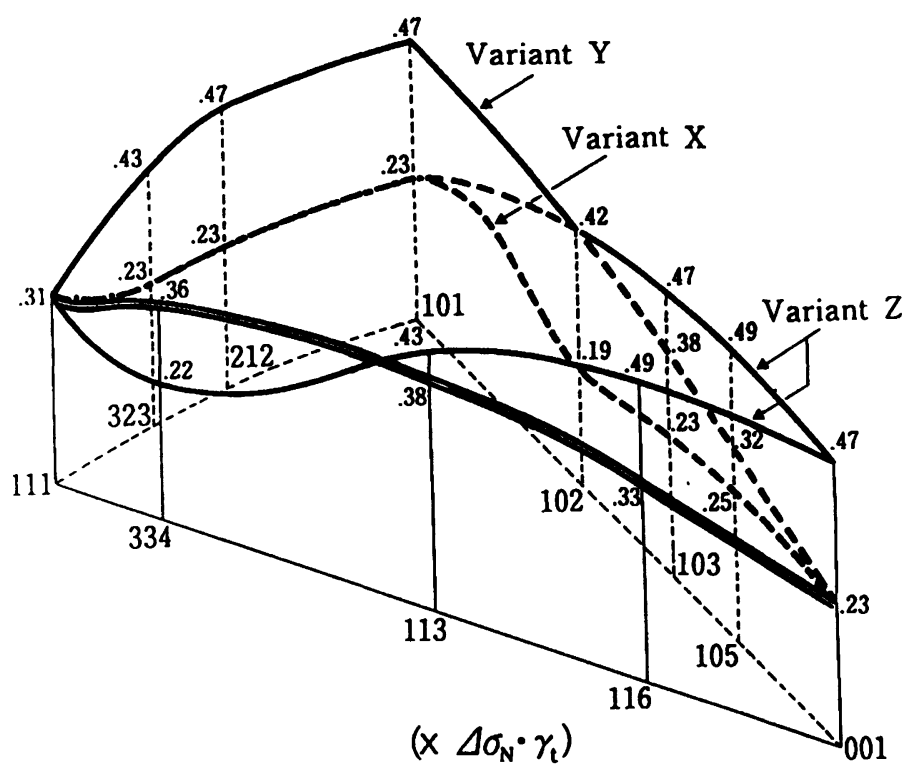

Figure 3 Effect of initial orientations on the work done by the diminished constraint stress against the shear strain in "Twinning Shear model" is calculated for each variant, and plotted on the stereographic triangle. 
Table 2 Effect of (001), (101) and (111) orientations on the selection of Bain variant. Bain variant consists of three components, that is, of variant $X$, variant $Y$ and variant $Z$, because the direction of $c$ axis (compression axis) can be taken parallel to either one of $X(T D), Y(R D)$ or $Z(N D)$ axis, respectively. The number of Bain variant which will be selected is indicated for each model

\begin{tabular}{c|c|c|c}
\hline \multirow{2}{*}{ Model } & \multicolumn{2}{|c|}{ Bain Strain model } & Twinning Shear model \\
\cline { 2 - 4 } & $\left|\varepsilon_{1}\right|>\left|\varepsilon_{2}\right|$ & $\left|\varepsilon_{1}\right|=\left|\varepsilon_{2}\right|$ & \\
\hline$\{001\}$ & 1 & 3 & 1 \\
$\{101\}$ & $3($ or 2$)$ & 3 & 1 \\
$\{111\}$ & 3 & 3 & 3 \\
\hline
\end{tabular}

It is seen from Figure 3 that the difference of the value of $\Delta \mathrm{W}$ between the selected 3 variants is maximum in (001) and (101): orientations and the variant selection will occur. On the other hand, the difference is minimum (zero) in (111); orientation and the variant selection will not occur. The results of them are showed in Table 2.

\section{EXPERIMENTAL VERIFICATION AND DISCUSSION}

As a result of calculation conducted in the previous section, it became clear that the maximum difference between BS and TS model appears when the tetragonal martensite with the Bain strain of $\left|\varepsilon_{1}\right|=\left|\varepsilon_{2}\right|$ forms in thin sheet of the $\{001\}_{\gamma}$ orientation (refer to Figure 2(b), Figure 3 and Table 2). That is, in this condition it is considered that all variants will form in BS model (variant is not selected) but only the component of variant $\mathrm{Z}$ will form in TS model.

To verify this, the following experiments have been conducted. At first Fe-30Ni alloy were melted with the induction vacuum furnace for the following reason:

(1) the cube texture ( $\{001\}$ orientation to the sheet plane) develops easily in $\gamma$ phase.

(2) Ms temperature lies near the R.T. and both $\gamma$ and $\alpha$ phase of before and after transformation can be obtained.

The ingot was hot rolled to $10 \mathrm{~mm}$ thick after the homogenization annealing. It was cold rolled severely to thin sheet of $0.15 \mathrm{~mm}$ thick. Then it was carburized at $900^{\circ} \mathrm{C}$ for $50 \mathrm{~h}$ and quenched into water to dissolve the carbon atoms as much as possible. The chemical composition of the specimen is shown in Table 1. Carbon did not dissolve in excess of $0.8 \%$ in this condition. Then the specimen was chemically polished, from the both side of the surfaces, to have reduced to the thickness of $30 \mu \mathrm{m}$. The specimen was subzeroquenched into the liquid nitrogen to induce martensitic transformation. Bain strain of this martensite in the as-formed "fresh state" was presumed that the $\varepsilon_{1} \doteqdot \angle 0.14 ; \varepsilon_{2} \fallingdotseq 0.11$ respectively according to the extensive and systematic study on martensite tetragonality of Fe-Ni-C alloys by Kajiwara and Kikuchi (1991). Though, this does not attain the desired Bain strain of $\left|\varepsilon_{1}\right|=\left|\varepsilon_{2}\right|$, but the result was not affected by the difference of this degree as seen later. Pole figures of austenite and martensite were measured on this specimen by $\mathrm{x}$-ray Schulz(reflection) or Decker (transmission) method, using Co target. Pole densities were shown by relative X-ray intensity with respect to the strongest $\mathrm{X}$-ray intensity and were indicated by arabic numerals in the pole figures. The (001) pole figure of austenite is shown in Figure 

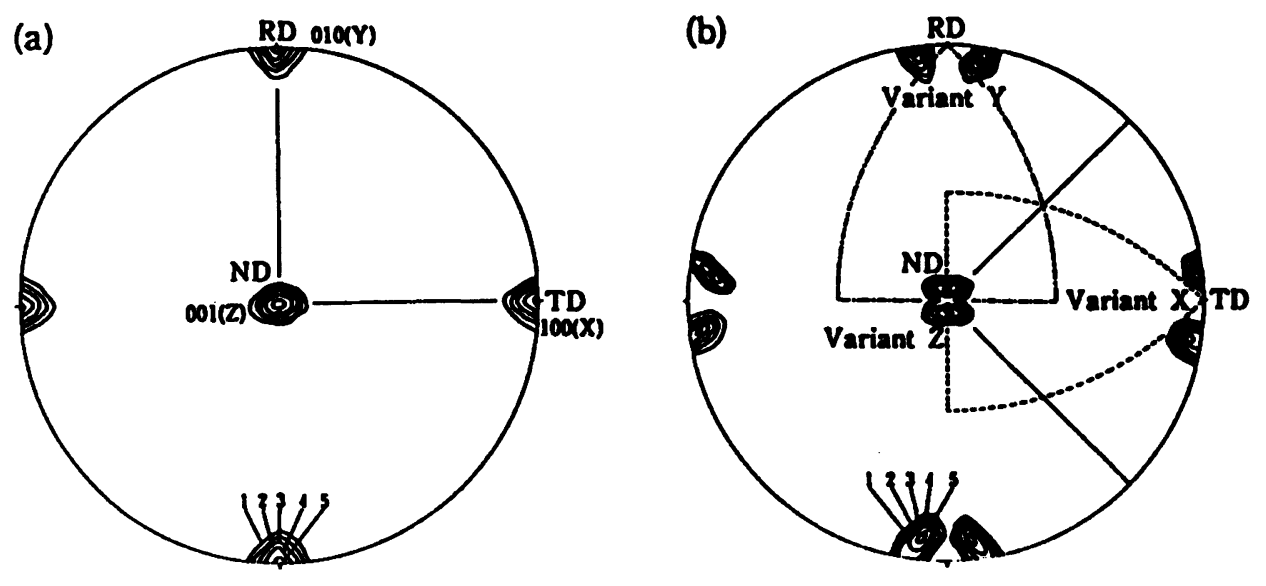

Figure 4 Pole figure of Fe-30Ni-O.8C alloy.

(a) $\{001\}$ pole of austenite, (b) pole of $c$ axis of tetragonal $\left(\left|\varepsilon_{1}\right| \fallingdotseq\left|\varepsilon_{2}\right|\right)$ martensite transformed from cube-textured austenite in (a). All variants were observed in it.

4(a). Austenite has well developed cube texture even after the carburization treatment. The pole of $c$ axis of the tetragonal martensite is shown in Figure 4(b).

Austenite lattice (face centered cubic structure) may also be described as a body centered tetragonal structure. There are three ways of choosing this tetragonal lattice in the austenite lattice, that is the direction of $c$ axis can be taken parallel to either one of $\mathrm{X}, \mathrm{Y}$, or $\mathrm{Z}$ axis respectively.

In Bain relation, each of them may serve as Bain compression axis i.e. rotation axis and transforms into $c$ axis of tetragonal martensite, which could be separated conveniently from $a$ and $b$ axis in the $x$-ray measurement. It is seen from the Figure 4(a) and Figure 4(b) that all of the three kinds of c axis of tetragonal martensite formed equally.

It became clear from the above results that the variant selection phenomenon in martensitic transformation coincided well with the prediction by BS model.

\section{CONCLUSIONS}

Variant selection processes without external stress were calculated on the BS and TS models, respectively and the experiments were conducted to examine the validity of the calculations. The results obtained are as follows:

(1) Variant selection phenomenon depends on the crystal orientation of the parent phase and on the Bain strain in the martensitic transformation.

(2) The effect of the anisotropic constraint stress on the shear deformation involved in the lattice change and on the Bain distortion appears most remarkably in the BCT martensite transformed from the cube textured parent phase.

(3) It became clear that such a variant that has the largest Bain strain component to the sheet normal, that is, the variant, of which the Bain distortion is prevented least of all by the anisotropic constraint stress, forms predominantly. 


\section{References}

1. Bain, E. C. (1924). Trans. AIME, 70, 25.

2. Bogers, A. J. and Burgers, W. G. (1964). Partial Dislocations on the \{110\} Planes in the B.C.C. Lattice and the Transition of the F.C.C. into the B.C.C. Lattice. Acta Metall., 12, 255-261.

3. Breedis, J. F. and Wayman, C. M. (1962), The Martensitic Transformation in Fe-31 wt Pct Ni. Trans. AIME, 224, 1128-1133.

4. Furubayashi, E. (1985a). Computer Simulation of Transformation Textures in Steels. Tetsu-toHagane, 71, 1155-1162.

5. Furubayashi, E. (1985b). Transformation Texture Analysis with Bain Relation-Monte Carlo Simulations. Tetsu-to-Hagane, 71, 1359-1366.

6. Furubayashi, E., Miyaji, H. and Nobuki, M. (1987). A Simple Model of Predicting the Transformation Textures in Thermomechanically Processed Steels. Trans. ISIJ, 27, 513-519.

7. Hashimoto, O. (1981). Eng. D. Thesis, Ôsaka University.

8. Higo, Y., Lecroisey, F. and Mori, T. (1974). Relation between Applied Stress and Orientation Relationship of $\alpha$ Martensite in Stainless Steel Single Crystals. Acta Metall., 22, 313-323.

9. Kajiwara, S. and Kikuchi, T. (1991). On the Abnormally Large Tetragonality of Martensite in Fe-Ni-C Alloys. Acta Metall. Mater., 39, 1123-1131.

10. Kurdjumov, G. and Sachs, G. (1930). Z. Phys, 64, 325.

11. Liu, W. P. and Bunge, H. J. (1991). Variant Selection in the Martensitic Transformation of an Fe-30\% Ni Alloy with Cube Texture. Materials Letters, 10, 336-343.

12. Miyaji, H. and Furubayashi, E. (1990). Effect of Specimen Size on the Variant Selection in Martensitic Transformation. Textures and Microstructures, 12, 189-197.

13. Miyaji, H. and Furubayashi, E. (1991). Effect of Stress on the Variant Selection in Martensitic Transformation. Textures and Microstructures, 14-18, 561-566.

14. Nishiyama, Z. (1934). Sci. Rep. Tohoku Univ., 23, 637.

15. Nishiyama, Z. (1936). Sci. Rep. Tohoku Univ., 25, 79. 